\title{
Colloidal Precursors from "Ball-Milling in Liquid Medium" Process for CuInSe, Thin Film
}

\author{
Jaehoon Chung and Seung-Joo Kim* \\ Department of Chemistry, Division of Energy Systems Research, Ajou University, Suwon 443-749, Korea \\ *E-mail: sjookim@ajou.ac.kr \\ Received June 18, 2010, Accepted July 19, 2010
}

Key Words: Solar cell, CuInSe 2 , Mechanosynthesis, Ball-milling, Solution deposition

$\mathrm{CuInSe}_{2}$ (CIS) and related chalcopyrite compounds are very promising materials for thin film solar cells due to their favorable band gap, high optical absorption coefficient and long-term stability. CIS-based solar cells have shown the highest conversion efficiency reaching a value of $20 \%$. ${ }^{1}$ However, the vacuumbased processes that are used to fabricate CIS thin-films have some drawbacks such as the complexity in process, high production cost and difficulty in scaling up. ${ }^{2}$ Recently, several research groups have proposed different non-vacuum deposition processes for CIS solar cell. ${ }^{3-6}$ For example, H. W. Hillhouse et al. prepared the CIS absorber layer by using "nanocrystal ink method" in which a colloidal nanocrystal ink was obtained from reaction of $\mathrm{CuCl}, \mathrm{InCl}_{3}$ and $\mathrm{Se}$ in oleylamine. ${ }^{3}$ D. B. Mitzi et al. used a solution-based precursor that was prepared by dissolution of $\mathrm{Cu}_{2} \mathrm{Se}, \mathrm{In}_{2} \mathrm{Se}_{3}, \mathrm{Ga}_{2} \mathrm{Se}_{3}$ and $\mathrm{Se}$ in hydrazine to fabricate the Ga-containing absorber layer, $\mathrm{Cu}(\mathrm{In}, \mathrm{Ga}) \mathrm{Se}_{2}{ }^{6}{ }^{6}$

Non-vacuum processes developed so far, however, have still limitations. The nanocrystal-ink method involves multi-step processes including separation and purification of the CIS particulates from impurity residues, otherwise the foreign elements caused by additives and suspension stabilizers may deteriorate the crystallinity and the electronic property of CIS layer. In the hydrazine solution method, the toxicity and corrosiveness of the hydrazine may cause an environmental problem as well as a decrease in durability of fabrication equipment. Considering the simplicity and safety in process for preparation of CIS precursor, mechanochemical synthesis deserves attention because the CIS compound can be directly formed via ball-milling the elemental $(\mathrm{Cu}$, In and $\mathrm{Se})$ reagents. In this process, the expected reaction is follows:

$$
\mathrm{Cu}+\mathrm{In}+2 \mathrm{Se} \rightarrow \mathrm{CuInSe}_{2} \quad \Delta H_{\mathrm{f}}=-204 \mathrm{~kJ} / \mathrm{mol}
$$

This reaction is self-propagating and completes in a short time because it is strong exothermic. ${ }^{7}$ To avoid agglomeration of the resultant powder, the mechanical milling process can be carried out in the presence of organic solvent. Very recently some attempts have been made to employ the wet ball-milling process in preparation of the CIS precursor. ${ }^{8-10}$ Up to now, however, systematic study concerning the effects of liquid medium on the ball-milled precursors for CIS has not been carried out. In this study, we compare the property of the colloids obtained under different wet conditions and propose a new effective route to prepare CIS thin film.

The effect of liquid medium in ball-milling process was assessed by observing the settling behaviors of as-prepared precursor suspensions. When the $\mathrm{Cu}, \mathrm{In}$, and Se powders were ball-milled in toluene (medium A), the products were coarse particles with poor dispersity in the medium as shown in Fig. 1. The mixture of pyridine and toluene (medium B) provided the precipitate with slightly increased dispersity compared to medium $\mathrm{A}$. In the case of medium $\mathrm{C}$ which is composed of ethylenediamine and toluene, the product becomes well dispersed and shows relatively good stability in the suspension. The best dispersion stability was observed in the product in the mixture of ethylenediamine and DMF (medium D) which showed no sedimentation at least a few hours. Fig. 2 shows the XRD patterns for the precipitates produced in each medium. The products in the medium A were $\mathrm{Cu}_{1-\mathrm{x}} \mathrm{In}_{\mathrm{x}}(\mathrm{x} \sim 0.2)$ alloy (JCPDS No. 657274) and CuInSe ${ }_{2}$ (JCPDS No. 87-2265), whereas single phasic $\mathrm{CuInSe}_{2}$ was formed in the medium B. In the ethylenediaminecontaining media (C and D), amorphous colloids were produced. From these observations the possible reactions in the liquid media are postulated as follows:

$$
\begin{aligned}
& (1-\mathrm{x}) \mathrm{Cu}+\mathrm{xIn} \rightarrow \mathrm{Cu}_{1-\mathrm{x}} \mathrm{In}_{\mathrm{x}} \\
& \mathrm{Cu}+\mathrm{In}+2 \mathrm{Se} \rightarrow \mathrm{CuInSe}_{2} \\
& \mathrm{Cu}+\mathrm{In}+2 \mathrm{Se}+\mathrm{xL} \rightarrow \mathrm{CuL}_{\mathrm{x}}^{+}+\left[\mathrm{InL}_{\mathrm{x}}\right]^{3+}+2\left[\mathrm{SeL}_{\mathrm{x}}\right]_{3}^{2-}
\end{aligned}
$$

(A)

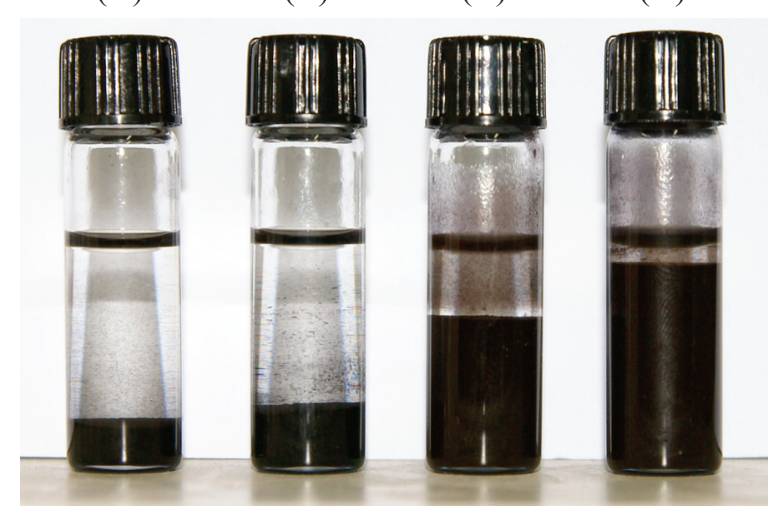

Figure 1. Dispersion nature of the precursors prepared using different liquid media: (A) toluene, (B) $2 \mathrm{M}$ pyridine in toluene, (C) $2 \mathrm{M}$ ethylenediamine in toluene, and (D) 2 M ethylenediamine in DMF. 


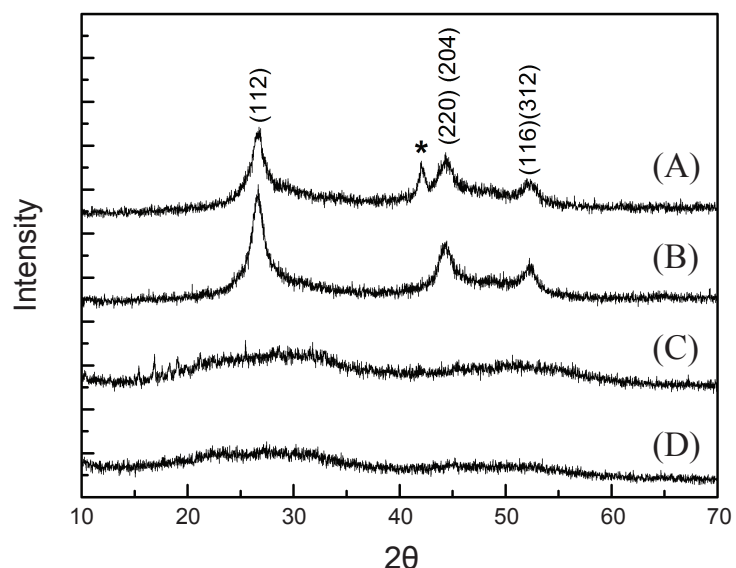

Figure 2. XRD patterns of the precursors prepared using different liquid media: (A) toluene, (B) $2 \mathrm{M}$ pyridine in toluene, (C) $2 \mathrm{M}$ ethylenediamine in toluene, and (D) $2 \mathrm{M}$ ethylenediamine in DMF. Asterisk denotes the $\mathrm{Cu}_{1-\mathrm{x}} \mathrm{In}_{\mathrm{x}}(\mathrm{x} \sim 0.2)$ phase.

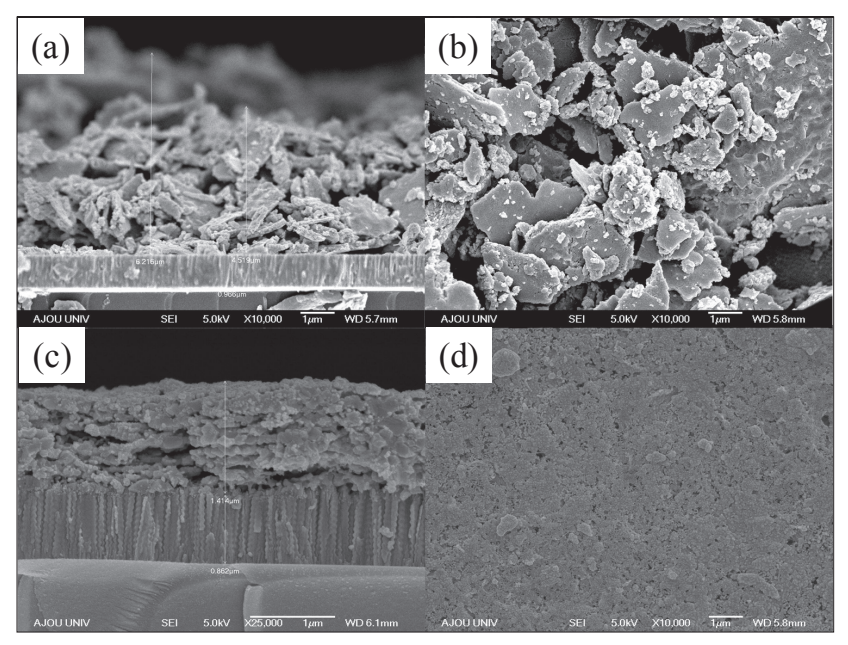

Figure 3. Cross-sectional and planner SEM images of the film after final heat treatment, (a) and (b) are corresponding to the film from the precursor using $2 \mathrm{M}$ pyridine in toluene (medium B). (c) and (d) are corresponding to the film from the precursor using $2 \mathrm{M}$ ethylenediamine in DMF (medium D).

Here, $\mathrm{L}$ is a pyridine or an ethylenediamine molecule. The inert medium such as toluene may hinder transferring the mechanical energy to individual reactant particles so that the formation of $\mathrm{CuInSe}_{2}$ (reaction 2) is not completed and the intermediate alloy $\mathrm{Cu}_{1-x} \mathrm{In}_{\mathrm{x}}$ remains (reaction 1). In the pyridinecontaining medium, pyridine may attack the metal surface to partially form a metal-pyridine complex, which increases the reactivity to form the monophasic $\mathrm{CuInSe}_{2}$. The formation of amorphous product in the ethylenediamine-containing media indicates that the metal-ethylenediamine complexation (reaction 3) can be the predominant reaction. Such strong complexation is attributed to the stronger basicity of ethylenediamine than that of pyridine. According to previous literature, amine reacts with metal chalcogenide to form an amine-metal-chalcogenide complex which is highly soluble in polar solvent. ${ }^{11}$ The medium D (the mixture of ethylenediamine and DMF) is more polar than the medium $\mathrm{C}$ (the mixture of ethylenediamine

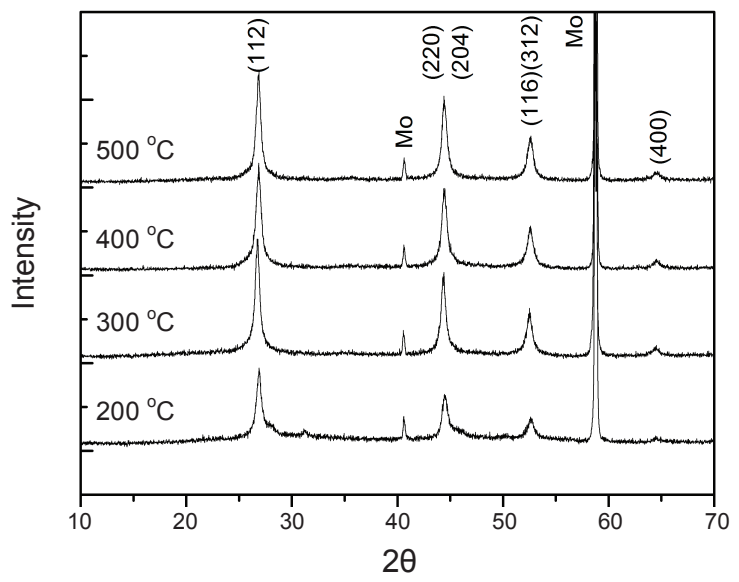

Figure 4. XRD patterns of the film prepared using $2 \mathrm{M}$ ethylenediamine in DMF (medium D) after heat treatment at different temperatures.

and toluene). Better dispersion in more polar solvent supports the formation of the metal-ethylenediamine and Se-ethylenediamine complexes.

Fig. 3 shows the morphologies for the CIS films made from different precursors. Although the precursor obtained in medium B showed the diffraction pattern corresponding to crystalline CIS compound, it provided a film with low quality due to a lot of cracks and pores (Fig. 3a and 3b). On the contrary, the amorphous colloidal precursor obtained in medium $\mathrm{D}$ leads to a relative dense film with regular thickness $(\sim 1.4 \mu \mathrm{m})$ as shown in Fig. $3 \mathrm{c}$ and $3 \mathrm{~d}$. Such comparison points out the importance of dispersion of the colloids in precursor rather than the crystallinity of the particulates in the coating process for CIS thin films. To use for CIS photo voltaic device, the CIS layer should be highly dense and of an appropriate thickness $(1 \sim 2 \mu \mathrm{m}) .{ }^{12}$ In this regard, the ball-milling process in medium $\mathrm{D}$ can be an effective way to prepare CIS layer. Fig. 4 shows a change in XRD pattern of the film made from the precursor $\mathrm{D}$ after heat-treatment at different temperature. The amorphous nature disappeared after heating at $200^{\circ} \mathrm{C}$. Above $300^{\circ} \mathrm{C}$, the XRD pattern of the film indicated the crystallization of chalcopyrite phase with most intense peaks at $2 \theta=26.9^{\circ}, 44.6^{\circ}$, and $52.8^{\circ}$, corresponding to (112), (220/204) and (116) planes, respectively. ${ }^{13-14}$ The lattice parameters are $\mathrm{a}=5.784(3) \AA$ and $\mathrm{c}=11.572(3) \AA$, which are well consistent with the previously reported values. ${ }^{15}$ The composition of the film obtained from heat treatment at $500{ }^{\circ} \mathrm{C}$ was confirmed with energy-dispersive $\mathrm{X}$-ray analysis. The ratio of atoms, $\mathrm{Cu}: \mathrm{In}: \mathrm{Se}=0.92: 1.03: 2.00$ is matched with the nominal composition $(1: 1: 2)$. Neither carbon nor oxygen element was not detected within experimental error range. Detailed studies of sintering process and the measurement of photovoltaic properties of the cells fabricated with the present CIS films are in progress.

Conclusively, CIS thin film can be fabricated by using the precursor obtained through ball-milling the elemental reagents in liquid media. The amorphous colloidal precursor with good dispersity was prepared in the medium that contains strong base and polar solvent (2 M ethylenediamine in DMF solution as used in this study). The "ball-milling in liquid medium" method 
requires only elemental sources as starting materials and a proper solution so that it can be employed without additional processes for separation and purification. As a simple and less-toxic preparative route, this method would be practically available to prepare CIS-related solar cells.

\section{Experimental Section}

Starting materials, elemental copper of $1 \mathrm{mmol}$, indium of $1 \mathrm{mmol}$ and selenium of $2 \mathrm{mmol}$ powders were loaded in a zirconium dioxide bowl $(20 \mathrm{~mL})$ with zirconium dioxide ball $(\mathrm{d}=3 \mathrm{~mm})$. The blended elemental mixtures were ball-milled in four different liquid media: medium $\mathrm{A}$ is toluene, medium $\mathrm{B}$ is $2 \mathrm{M}$ pyridine in toluene, medium $\mathrm{C}$ is $2 \mathrm{M}$ ethylenediamine in toluene, and medium $\mathrm{D}$ is $2 \mathrm{M}$ ethylenediamine in dimethylformamide (DMF). The volume of each liquid medium was $6 \mathrm{~mL}$. Every ball-milling was carried out at $700 \mathrm{rpm}$ for $12 \mathrm{~h}$ using Planetary micromill ("pluverisette 7", Fritsch).

Spin coatings were performed by flooding a Mo/glass substrate surface with the prepared precursors and spinning at 2000 rpm for $180 \mathrm{sec}$ after $1000 \mathrm{rpm}$ for $15 \mathrm{sec}$, which is sufficient to cover the substrate with uniform thickness $\sim 0.2 \mu \mathrm{m}$ at once. The deposited films were then preheated at $290{ }^{\circ} \mathrm{C}$ during $5 \mathrm{~min}$ under Ar atmosphere. To achieve desired film thickness, this process was sequentially repeated $8 \sim 10$ deposition cycles. Final heating of the films was performed in the Ar-filled quartz reactor at $500{ }^{\circ} \mathrm{C}$ for $30 \mathrm{~min}$.

The phase of the prepared films were investigated by X-ray diffraction (Rigaku, D/MAX-2500), using $\mathrm{Cu}$ K $\alpha$ line. The composition and morphology were identified with field emission scanning electron microscope (JEOL, JSM-6700F) and energy dispersive spectroscopy (Genesis apex, EDAX).

Acknowledgments. This research was partly supported by the Converging Research Center Program (2009-0093701) and Priority Research Centers Program (2009-0094047) through the National Research Foundation of Korea (NRF) funded by the Ministry of Education, Science and Technology.

\section{References}

1. Repins, I.; Contreras, M. A.; Egaas, B.; DeHart, C.; Scharf, J.; Perkins, C. L.; To, B.; Noufi, R. Prog. Photovoltaics Res. Appl. 2008, 16, 235.

2. Eberspacher, C.; Fredric, C.; Pauls, K.; Serra, J. Thin Solid Films 2001, 387, 18 .

3. Hou, W. W.; Bob, B.; Li, S.; Yang, Y. Thin Solid Films 2009, 517, 6853.

4. Guo, Q.; Kim, S. J.; Kar, M.; Shafarman, W. N.; Birkmire, R. W.; Stach, E. A.; Agrawal, R.; Hillhouse, H. W. Nano Lett. 2008, 8, 2982.

5. Panthani, M. G.; Akhavan, V.; Goodfellow, B.; Schmidtke, J. P.; Dunn, L.; Dodabalapur, A.; Barbara, P. F.; Korgel, B. A. J. Am. Chem. Soc. 2008, 130, 16770.

6. Mitzi, D. B.; Yuan, M.; Liu, W.; Kellock, A. J.; Chey, S. J.; Deline, V.; Schrott, A. G. Adv. Mater. 2008, 20, 3657.

7. Wada, T.; Kinos, H. J. Phys. Chem. Solids 2005, 66, 1987.

8. Wada, T.; Matsuo, Y.; Nomura, S.; Nakamura, Y.; Miyamura, A.; Chiba, Y.; Yamada, A.; Konagai, M. Phys. Stat. Sol. (a)203. 2006, 11, 2593.

9. Kubo, J.; Matsuo, Y.; Wada, T.; Yamada, A.; Konagai, M. Mater. Res. Soc. 2009, 1165, M05-13.

10. Vidhya, B.; Velumani, S.; Arenas-Alatorre, J. A.; Morales-Acevedo, A.; Asomoza, R.; Chavez-Carvayar, J. A. Mater. Sci. Eng. $B$ 2010, article in press.

11. Mitzi, D. B. Inorg. Chem. 2005, 44, 3755.

12. Todorov, T.; Mitzi, D. B. Eur. J. Inorg. Chem. 2010, 17.

13. Ahn, S. J.; Kim, K. H.; Chun, Y. G.; Yoon, K. H. Thin Solid Films 2007, 515, 4036

14. Suryanarayana, C.; Yoo, S. H.; Groza, J. R. J. Mater. Sci. Lett. 2001, 20,2179

15. Wada, T.; Kinoshita, H.; Kawata, S. Thin Solid Films 2003, 431, 11 . 